\title{
Towards improving e-government services using social media-based citizen's profile investigation
}

\author{
Ameni Kacem \\ LARODEC, ISG - IRIT, Toulouse \\ Tunis University, Tunisia \\ Université Paul Sabatier, France \\ ameni.kacem@gmail.com \\ Haithem Ghorbel \\ LARODEC, ISG \\ Tunis University, Tunisia \\ gho.haithem@gmail.com
}

\author{
Rami Belkaroui \\ LARODE C, ISG - LINA, Nantes \\ Tunis University, Tunisia \\ Université de Nantes, France \\ rami.belkaroui@gmail.com \\ Rim Faiz \\ LARODEC, IHEC Carthage \\ Carthage University, Tunisia \\ rim.faiz@ihec.rnu.tn
}

\author{
Dhouha Jemal \\ LARODEC, ISG \\ Tunis University, Tunisia \\ dh.jemal@gmail.com
}

\author{
Ines Hammami Abid \\ Business School IAE de Nice, \\ Sophia Antipolis University, France \\ hammami.abid@gmail.com
}

\begin{abstract}
Governments are making considerable efforts in order to enhance citizens' participation in their decision-making and policy processes. In order to understand the citizens' needs, the decision makers need to know more about citizens' behaviors, preferences and ability to use e-government online services. Recently, social media represents a strategic opportunity that can help governments to become more transparent by providing citizens with better services and enhancing information's access in a way that makes them more involved.

In this paper, we propose a new conceptual framework that investigates users and communities' profiles based on social media. The aim of this paper is to explore the social citizens' profiles in order to improve and adapt public services, decision making, information sharing, transparency and collaboration enhancing...to the individual's current and relevant needs.
\end{abstract}

\section{CCS Concepts}

- Information systems $\rightarrow$ Information retrieval $\rightarrow$ Users and interactive retrieval $\rightarrow$ Personalization

\section{Keywords}

e-government, social media, big data, user profile, recommendation, open data

\section{INTRODUCTION}

E-government refers to the use of information and communication technologies (ICTs) to achieve citizens' satisfaction through more suitable services. Governments need to be more interactive and reach citizens where they are. Therefore, e-government is considered as one of the channels to enhance public trust in the government through increased accountability and empowerment

SAMPLE: Permission to make digital or hard copies of all or part of this work for personal or classroom use is granted without fee provided that copies are not made or distributed for profit or commercial advantage and that copies bear this notice and the full citation on the first page. To copy otherwise, or republish, to post on servers or to redistribute to lists, requires prior specific permission and/or a fee. of its citizens [4].

Recently, social media services have become the main way of communication, content sharing and opinion expressing and is considered as an invaluable source that help collect all needed information about citizens and communities. In fact, governments can take advantage of the high capabilities and popularity of social media, in order to improve government's strategies and performance. The considerable massive interest and involvement on social networks use reveals that there is now serious potential for the enhancement of online participation. A plausible indicator of this potential is the rising number of those using one or more online government services, which has increased from $39 \%$ in 2005 to $57 \%$ in 2011 [5].

Social media have already played seminal roles in political protests in various countries, including Tunisia, Iran, Egypt and others caught up in the Arab Spring revolutions. The Arab Spring is probably the most recent example when talking about social media abilities to impact government and shape political discourse. Moreover, social media represents a strategic opportunity that should be cautiously managed to better engage individuals, businesses and public organizations [6]. It can help government to become more transparent by providing citizens with better services and information's access, opening an active communication channel, in a way that makes them more involved. In this paper, we address the following questions: How can egovernment systems exploit data provided by social media? And how those data contribute to improve efficiency of e-government services and government decision-making process?

Thus, we propose a new conceptual framework that investigates users and communities' profiles based on social media. It allows a better delivery of services to citizens, an increased transparency and a greater convenience.

The remainder of this paper is organized as follows: we begin by describing some related works. In section 3, we present our conceptual framework of citizens' profile investigation based on social media for e-government services improvement. Finally, we present a summary of our work and some future directions.

\section{RELATED WORK}

In this section, we review prior solutions in the e-government field using social media, user profiles and Big Data techniques. 


\subsection{User profile and e-government}

Personalization of Information Retrieval (IR) systems in the egovernment field is used in order to better satisfy citizens' needs, through providing personalized public services or helping citizens find relevant information within the huge rising amount of data. Most of the prior work that address the user-profiling problem in e-government does not include activities of the user extracted from social media. They rather use the demographic information, user browsing history or feedback. However, the user's profile content can be extracted from his online activities on social media Web sites. In fact, recently IR systems take advantage of the emergence of user-created content such as blogs, microblogs and social networks [7]. This kind of content is not commonly used in the e-government field.

Many prior works use the user profile explicitly extracted from his inputs when interacting or creating his/her account on public services platforms. For instance, in [8], the authors propose a personalization platform that adapt proposed services to each user profile. After the creation of the user profile from multiple sources, they used two types of profiles: a long-term profile that contains static information about the user, and a short-term profile that considers only information extracted from the current session.

More recent works exploit social media as a source of government services' personalization such as the work proposed by [3] in which the authors explained the importance of having a human interface with citizens that can be obtained thanks to social media especially forums. The work of [13] highlighted that social media is bringing new elements to the electronic government such as the exploration of online communities, social networking and user generated content. They consider that the collaborative aspect is very important in the Web 2.0 because it integrates communication, interaction, content sharing and social networking.

\subsection{E-Gove rnment between Open and B ig Data}

When combined with the power of social media, Open and Big Data offer substantial opportunity to foster civic engagement and social innovation. Open Data, also referred as open Public Sector Information (PSI), has received considerable attention in recent years in order to increase transparency, participation and government efficiency. In [9], the authors present Open Data as a recent phenomenon in an early stage of development. They analyzed the benefits and barriers of Open Data systems by synthesizing people's experiences with Open Data.

The promises and potential of Big Data in transforming digital government services, governments, and the interaction between governments, citizens, and the business sector, are substantial through increased efficiency and effectiveness in the delivery of services. As described in [12], the adoption and benefits of Big Data solutions in various government projects can support the government's decision-making processes for ushering in a new era of digital government services.

In [2], the authors reviewed and analyzed the U.S. policy context regarding Big Data. They present some examples of Big Data initiatives that offer views of the promise of Big Data-driven application and research, such as a range of Big Data techniques in order to assess implications of climate change on the New York state, and provide strategies to deal with this issue in areas such as agriculture, public health, energy, and transportation. In this paper, we study how we can learn from the presented experiences, and create solutions that better meet citizens' needs using some of today's Big Data applications.

\section{THE PROPOSED CP-GOV FRAMEWORK}

Aiming to improve and adapt public services, decision making, information sharing, transparency and collaboration with citizens, we propose a conceptual framework of citizens' profile investigation for e-government services improvement, called CPGov, as depicted in Figure 1. This framework is comprised of six main components assembled to offer the most relevant recommendations that meet citizens' requirements. It can be applied to various government departments, such as education, tourism, welfare, and healthcare departments. The CP-Gov framework offers two facets of relevant information for citizens and policy makers.

- For citizens: the personalized recommendations are offered to users according to their profiles to improve the quality of public service delivery.

- For policy makers: our framework offers citizens' needs based on profile investigation, in order to improve and adapt decision making, e-government strategies, transparency and collaboration.

In the following, we will give a general description of each main component focusing on conceptual functionality and technical details.

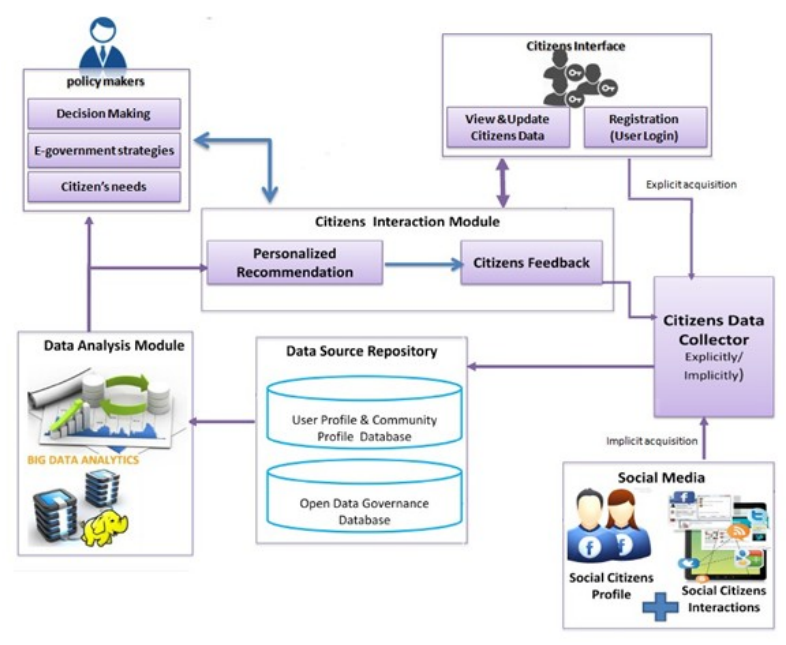

Figure 1. Our CP-Gov proposed framework.

\subsection{Personalized Citizens Interface and Data Collector}

The personalized citizen interface ensures the interaction between citizens and the system. The login of each citizen in the governmental framework is ensured by providing his or her user name and password obtained upon registration. The citizen personal information is gathered explicitly when she makes registration such as their demographic data (name, age,..., personal interests and preferences towards e-government services). In fact, the user has the possibility to update regularly these information in database through her personal page. Implicitly, information related to context (personal and social) are 
captured from citizens' social profile and activities on social media.

\subsection{Social Media-Based Citizens Profiles Construction}

The profiling process, in our CP-GOV, is composed of two main elements. The first one is the citizen profile creation using his activities in social media websites: Social Citizens Profile (SCP). The second one called Social Citizens Interactions (SCI) resides in integrating citizens' interactions in the profile.

\subsubsection{Social Citizens Profiles (SCP)}

Considering the social media emergence, users in the social networking sites express freely their opinions, preferences and share content in which they are interested. In our approach, we first extract citizens' activities on social media specifically Facebook as it is the most popular social media used by citizens especially in Tunisia.

For user profiling construction, we use the keyword-based representation where the keywords can be explicitly provided from each user or implicitly extracted from his online activities on social media. Formally the user profile is presented as follows:

$$
\overrightarrow{U_{m_{j}}}=\left(t_{1}: W_{1}^{m_{j}}, t_{2}: W_{2}^{m_{j}}, \ldots t_{n}: W_{n}^{m_{j}}\right)
$$

where: $t$ is the extracted term, $W$ is its corresponding weight and $m_{j}$ design the publication moment. After collecting terms that are used in our profiling approach, we assign in each day the normalized term frequency (nTF) score that gives an overview of the terms' frequency distribution. Thus, considering the relative frequency of a term $f\left(t_{j}\right)$ in a publication moment $m_{j}$ compared to the frequencies of all terms $\sum f\left(t_{k}\right)$, we obtain the following content feature:

$$
\operatorname{Cont}_{F}=n T F=\frac{f\left(t_{i}\right)^{m_{j}}}{\sum f\left(t_{k}\right)^{m_{j}}}
$$

Owing to the constantly changes in the users' preferences, we use the day as a temporal unit. We use the same weighting scheme as in [11] where we combine short-term and long-term profiles by integrating the Kernel Gaussian function in order to give fresh terms higher value:

$$
\operatorname{Temp}_{F}=K\left(m_{c}, m_{j}\right)=\frac{1}{\sqrt{2 . \pi} \cdot \sigma} \cdot \exp \left[\frac{-\left(m_{o} m_{j}\right)^{2}}{2 . \sigma^{2}}\right]
$$

where $\sigma$ is the interpolation coefficient, $m_{c}$ is the current moment and $m_{j}$ is a prior moment of publishing a post on Facebook. Thus, the final score $\mathrm{W}$ is calculated according to the following formula:

$$
W=\sum \text { Cont }_{F} \cdot \text { Temp }_{F}
$$

Where Cont $_{F}$ and $\operatorname{Temp}_{F}$ design respectively Content-Feature and Temporal-Feature presented previously. Consequently, our weighting scheme supports both the frequency and the time distributions.

\subsubsection{Social Citizens Interactions (SCI)}

In order to enrich the created user profile, we propose to integrate social citizens' interactions. In order to efficiently integrate citizens' interactions, we first leverage the "hashtags" of Facebook status in order to extract the present entities. Specifically, we consider each hashtag in Facebook posts as a single entity. Then we retrieve a common set of conversation using our conversations trees detection system [1] that covers these entities. In the second step, we analyze all of the conversation for all of the discovered entities in a user's set of Facebook posts, and determine the set of categories that define that user's topic profile (e.g., the topics of interest). To generate topic profile, we rank each of the categories using a frequency ranking function. After that, we define topic profile as the top-K categories, ranked according to our ranking function. The output of this step is used to enrich the user and community profiles by boosting the interests and preferences that meet the top-K categories of topic profiles.

\subsubsection{Community Profiling}

Based on citizens' information collected from social media, user communities can be formed to represent groups of users who share common characteristics, interests and/or needs in a specific e-government domain. This component can be used to predict and recommend the most relevant services among the available ones. Relevant services will be generated for each citizen community as personalized output based on interests and needs determin ed from citizens' profiles, in order to increases citizens satisfaction levels.

\subsection{Data Source Repository (DSR)}

The role of the Data Source Repository component is to store all the data that is required as an input for the data analy sis module. It comprises of:

- User Profile Database: It stores built citizens' profiles based on the implicit and explicit collected information. The purpose of storing the profiles is to make use of them as input for the next phase of analysis in order to capture the needs, goals, values, expectations and habits of users into well-defined user groups.

- Open Data Governance Database: It contains data collected on the provision of open data initiatives. It includes all the public services that we aim to recommend as well as their features and rules. In fact, taking advantage for data available on national portals, can enabling more effective interactive and collaborative governance.

The DSR component is expected to support huge volume of data: citizens' collected information from social media, citizens' input information and available government data. This requires reliable technology and solution to handle with the massive quantities of data. It is not just about storage of and access to data, it is necessary to think about data security, integrity and availability [10]. In our framework, Hadoop has been chosen as the solution to answer our need for a powerful and trusted storage technology. Indeed, this solution can ensure our data security in the face of faults and failures in disks, nodes and networks.

\subsection{Data Analysis Module}

CP-GOV is based on the emergence of network access, citizens' personal information that become available especially on social media reflecting their needs, goals, values, expectations and habits. Due to the huge volume and the unstructured nature of those data, we use Hadoop and its data analysis system called MapReduce that is characterized by performing heavy processing on large structured and unstructured data volumes. In fact, when the data regard ing user and communities profiles are load ed on the cluster, they are broken down into smaller blocks and distributed throughout the cluster in order to enable a Hadoop MapReduce system of processing many parts of them in parallel by executing map and reduce functions on smaller subsets.

\subsection{Citizens Interaction Module}

In order to increase citizen satisfaction levels, the Citizens Interaction Module (CMI) consists on automatically recommending the most relevant services to a given citizen. We use a content-based filtering ap proach. First, each citizen's profile 
is analyzed as it contains his/her preferences and properties described in section 3.2. Second, the similarity between the user's profile and each service, which is also represented as a vector of weighted keywords, is measured. The user-service similarity can be obtained using the cosine measure for instance. Then, the recommendations are generated and presented through notification to citizens as a top-N list of services that have the most important similarity and consequently fit the best the users' profiles and needs. Finally, direct feedback from a citizen, in the form of a like or dislike button, can be used to assign higher or lower value to certain services and consequently contributes to maintain recommendation quality and consistency.

\subsection{Policy Makers}

Through our CP-Gov framework, we aim to help policy makers and government organizations improve their strategies. Thus, output rep orts are available using data mining techniques based on users and communities profiles, their preferences and demographic information. Those reports help not only to improve efficiency and effectiven ess of services delivery, but also to define long-term strategies, to adjust policies and national projects development, and to welcome new ideas and ways of collaboration with citizens.

\section{CONCLUSION}

Conscious of social media opportunities and of the government need to enhance public transparency, interactivity and accountability, we find that it is important to develop solutions for this issue. In this context, a conceptual framework for boosting interaction government-citizens is proposed. The CP-Gov framework was designed in the aim of offering personalized egovernment services to citizens. For the government side, the framework might improve its decision-making process by means of better understanding citizens' needs and expectations. In future work, we will focus on evaluating and validating our CP-Gov framework in a particular field, and try to develop a functional system to provide the personalized e-government services to citizens.

\section{REFERENCES}

[1] R. Belkaroui, R. Faiz, and A. Elkhlifi. 2015. Social users interactions detection based on conversational aspects. In New Trends in Intelligent Information and Database Systems, 598 of Studies in Computational Intelligence (2015), Springer International Publishing,161-170.

[2] J. C. Bertot and H. Choi. 2013. Big data and e-government: issues, policies, and recommendations. In Proceedings of the 14th Annual International Conference on Digital Government Research (Quebec City, QC, Canada, June 17 20, 2013). DGO'13. ACM, New York, NY, 1-10. DOI $=$ http ://doi.acm.org/10.1145/2479724.2479730
[3] S. K. Bista, S. Nepal, and C. Paris. 2013. The human touch of government services. In Late-Breaking Results, Project Papers and Workshop Proceedings of the 21st Conference on User Modeling, Adaptation, and Personalization (Rome, Italy, June 10-14, 2013). UMAP'13. Springer International Publishing.

[4] C. C. Demchak, C. Friis, and T. M. LaPorte. 2000. Webbing governance: National differences in constructing the public face. Handbook of public information system. 2, 155 (2000).

[5] W. H. Dutton and G. Blank. 2011. Next generation users: the internet in Britain. Oxford Internet Survey 2011. Oxford Internet Institute, University of Oxford.

[6] S. Hofmann, D. Beverungen, M. Räckers, and J. Becker. 2013. What makes local governments' online communications successful? Insights from a multi-method analysis of Facebook. In GOV INFORM Q 30,4 (Oct.2013), 387-396.

[7] Y. Hussain. 2014. Social Media as a Tool for Transparency and Good Governance in the Government of Gilgit-Baltistan, Pakistan. In: Crossroads Asia Working Paper Series, No. 22.

[8] I.Zaoui, H. Elmaghraoui, D. Chiadmi, and L. Benhlima. 2014. Towards a personalized e-government platform. In International Journal of Computer Science: Theory and Application 2,2 (2014), 35-40.

[9] M. Janssen, Y. Charalabidis, and A. Zuiderwijk.2012. Benefits, adoption barriers and myths of open data and open government. In Information Systems Management 29 (2012), 258-268

[10] D. Jemal and R. Faiz. 2015. What if mixing technologies for Big Data mining and queries optimization. In Proceedings of the 7th International Conference on Computational Collective Intelligence, Technologies and Application (Madrid, Spain, September 23-23, 2015). ICCCI 2015. Springer International Publishing, 619-627.

[11] A. Kacem, M. Boughanem, and R. Faiz. 2014. Timesensitive user profile for optimizing search personalization. In User Modeling, Adaptation, and Personalization 8538 (2014), Springer International Publishing, 111-121.

[12] G.-H. Kim, S. Trimi, and J.-H. Chung. 2014. Big-Data Applications in the Government Sector. Commun. ACM 57,3 (Mar.2014), 78-85. DOI=http://doi.acm.org/10.1145/2500873

[13] C. G. Reddick. 2010. Citizens and E-government: Evaluating Policy and Management. IGI Global. 